\title{
EXISTENCE OF TRAJECTORIES WITH UNBOUNDED CONSUMPTION FOR A MODEL WITH INNOVATIONS
}

\author{
ALEXANDER J. ZASLAVSKI
}

Received 7 March 2004; Accepted 26 September 2004

We consider a model of economic dynamics with discrete innovations and establish the existence of trajectories with unbounded consumption.

Copyright (c) 2006 Hindawi Publishing Corporation. All rights reserved.

\section{Introduction}

We consider the model with discrete innovations introduced in [1] and studied in [2-9]. In this model the state of the economy is determined by a set of operating technologies, a collection of funds corresponding to these technologies, and a set of known, but as yet not implemented technologies. To introduce a new technology, expenditures of the already utilized types of funds are required. As a result of these expenditures, the new technology at the next instant of time will be introduced into action with a certain initial reserve of new funds.

We consider a single-product economy which deals with two production factors: labor $L$ and funds $K$. The time is assumed to be discrete and the amount of labor is constant and equal to unity. The state of the economy is determined by a set of operating technologies, a collection of funds corresponding to these technologies and a set of known, but as yet not implemented technologies.

A technology is a pair $(f, v)$ where $f$ is a production function of two variables $K, L$ and $v \in[0,1)$. Possessing at time $t$ funds $K$ and labor resources $L$, the economy utilizing the technology $(f, v)$ will produce during a unit time interval, a product in the amount of $f(K, L)$. Moreover, at time $t+1$ the economy will still have in its possession the used old funds in the amount of $v K$.

To introduce a new technology, expenditures of the already utilized types of funds are required. As a result of these expenditures, the new technology at the next instant of time will be introduced into action with a certain initial reserve of the new funds.

Let $I=\{0,1, \ldots\}$ and $\left\{\left(f^{i}, v^{i}\right): i \in I\right\}$ be the set of all technologies which can be utilized in the production process. At time $t \in I$ the state of the economy is given in the form

$$
\left(I_{0}^{t}, I_{n}^{t},\left(K_{t}^{i}, C_{t}^{i}\right)\left(i \in I_{0}^{t}\right)\right) \text {, }
$$

Hindawi Publishing Corporation Journal of Inequalities and Applications Volume 2006, Article ID 34380, Pages 1-11 DOI 10.1155/JIA/2006/34380 
where $I_{0}^{t}$ is a finite set of numbers (indices) of technologies introduced by the time $t, I_{n}^{t}$ is the set of numbers of technologies which are available in principle but not introduced, and $K_{t}^{i}, C_{t}^{i} \geq 0$ are the funds and consumption of the $i$ th type available at time $t$ which correspond to the technology with the number $i$. We will assume that at time $t$, the following information is available:

$$
\left(K^{i}\right), \quad\left(s^{i j}\right) \quad\left(i \in I_{n}^{t}, j \in I_{n}^{t} \cup I_{0}^{t}\right),
$$

where $s^{i j} \geq 0$ is the expenditure of the $j$ th funds required for introduction of the $i$ th technology and $K^{i}>0$ is the initial amount of the $i$ th fund which is obtained at the initial time of utilization of the $i$ th technology. At time $t+1$ the economy may pass over to the state

$$
\left(I_{0}^{t+1}, I_{n}^{t+1},\left(K_{t+1}^{i}, C_{t+1}^{i}\right)\left(i \in I_{0}^{t+1}\right)\right)
$$

for which

$$
\begin{gathered}
I_{0}^{t} \subset I_{0}^{t+1} \subset I_{0}^{t} \cup I_{n}^{t}, \\
I_{0}^{t+1} \backslash I_{0}^{t} \subset\left\{i \in I_{n}^{t}: s^{i j}=0 \forall j \in I_{n}^{t}\right\}
\end{gathered}
$$

and the numbers $L_{t}^{i} \geq 0, i \in I_{0}^{t}$ are determined such that

$$
\begin{gathered}
\sum_{i \in I_{0}^{t}} L_{t}^{i} \leq 1, \\
K_{t+1}^{j} \geq v^{j} K_{t}^{j} \quad \forall j \in I_{0}^{t}, \\
K_{t+1}^{i}=K^{i}, \quad C_{t+1}^{i}=0, \quad i \in I_{0}^{t+1} \backslash I_{0}^{t}, \\
K_{t+1}^{j}-v^{j} K_{t}^{j}+C_{t+1}^{j}+\sum_{i \in I_{0}^{t+1} \backslash I_{0}^{t}} s^{i j} \leq f^{j}\left(K_{t}^{j}, L_{t}^{j}\right) \quad \forall j \in I_{0}^{t} .
\end{gathered}
$$

(We assume here that the result of a summation over an empty set equals zero.) Note that in the model under consideration the newly produced product is used for consumption and expenditures related to an introduction of new technologies. Sometimes the state of the economy at time $t$ will be written in the form

$$
\left(I_{0}^{t}, I_{n}^{t},\left(K_{t}^{i}, C_{t}^{i}, L_{t}^{i}\right)\left(i \in I_{0}^{t}\right)\right)
$$

where $\left(L_{t}^{i}\right)\left(i \in I_{0}^{t}\right)$ is the distribution of labor resources at time $t$. We do it in the case when some description of this distribution is required. When describing a trajectory of the model we would also include into its description the corresponding sequence of distributions of labor resources, most often only in the case when some information about these resources is required. However, in any case a definite sequence of distributions of labor resources is always associated with a trajectory of the model.

Denote by $R_{+}^{l}$ the cone of elements of the Euclidean space $R^{l}$ with nonnegative coordinates. Below all the technologies under consideration $(f, v)$ will assume to be such that $f: R_{+}^{2} \rightarrow R_{+}$be a continuous, superlinear (superadditive, positively homogeneous) 
function,

$$
\begin{gathered}
f(0,1)=f(1,0)=0, \\
f(x, 1)<f(\lambda x, 1)<\lambda f(x, 1) \text { for each } \lambda>1 \text { and each } x>0,
\end{gathered}
$$

and there exists $X \in R_{+}$such that $f(1, X)>1-v$.

Let $(f, v)$ be a technology. It is easy to see that there exists a unique number $x(f, v)>0$ such that

$$
f(x(f, v), 1)=(1-v) x(f, v) .
$$

For $x_{0}>0$ the inequality $f\left(x_{0}, 1\right)>(1-v) x_{0}$ holds if and only if $x_{0}<x(f, v)$ and the sequence

$$
x_{t}=v x_{t-1}+f\left(x_{t-1}, 1\right), \quad t=1,2, \ldots
$$

converges to $x(f, v)$ as $t \rightarrow \infty$. Evidently $x(f, v)$ is a characteristic of the technology $(f, v)$ which evaluates its production capabilities.

The technology $(f, v)$ is associated with a dynamic model of the economy whose trajectory is a sequence $\left(K_{t}, C_{t}\right), t=0,1, \ldots$, where $K_{t}, C_{t} \geq 0$ are the funds and consumption available at time $t$ which satisfy

$$
\begin{aligned}
K_{t+1}-v K_{t} & \geq 0 \\
K_{t+1}-v K_{t}+C_{t+1} & \leq f\left(K_{t}, 1\right)
\end{aligned}
$$

for all $t=0,1, \ldots$

It is easy to see that for any model trajectory $\left(K_{t}, C_{t}\right), t=0,1, \ldots$ we have

$$
\begin{gathered}
\limsup _{t \rightarrow \infty}\left(v K_{t}+f\left(K_{t}, 1\right)\right) \leq x(f, v), \\
\limsup _{t \rightarrow \infty} K_{t} \leq x(f, v) .
\end{gathered}
$$

Moreover, for any initial state of the model $\left(K_{0}, C_{0}\right)$ with $K_{0}>0$ there exists a trajectory $\left(K_{t}, C_{t}\right), t=0,1, \ldots$ such that $K_{t} \rightarrow x(f, v)$ as $t \rightarrow \infty$.

Let $X=\left(K_{t}, C_{t}\right)(t \in I)$ be a model trajectory. Set

$$
w(X)=\limsup _{T \rightarrow \infty} T^{-1} \sum_{t=0}^{T-1} C_{t} .
$$

Evidently $w(X) \leq x(f, v)$. Set

$$
w(f, v)=\sup \{w(X): X \text { is a model trajectory }\} .
$$

The number $w(f, v)$ is a characteristic of the technology $(f, v)$ which evaluates its consumption capabilities.

It is easy to verify that the following result is true. 
4 Existence of trajectories

Proposition 1.1. There exists a number $h(f, v) \in(0,1)$ such that

$$
\begin{gathered}
\lim _{x \rightarrow \infty}(h(f, v) f(1, x)) \in(1-v, \infty], \\
(1-h(f, v)) f(x(h(f, v) f, v), 1)=w(f, v)>0 .
\end{gathered}
$$

\section{The main result}

Consider the model with discrete innovations introduced in Section 1. We assume that for each $i, j \in I$

$$
s^{i j}>0 \quad \text { if and only if } i=j+1
$$

and that

$$
s^{(i+1) i}<f^{i}\left(x\left(f^{i}, v^{i}\right), 1\right)
$$

for each $i \in I$.

Remark 2.1. Let $i \in I$ and the $i$ th technology be introduced at time $t_{0}$. Assume that $K^{i}<$ $x\left(f^{i}, v^{i}\right)$. This inequality means that the initial amount of the $i$ th fund is less than the characteristic of the technology $\left(f^{i}, v^{i}\right)$ which evaluates its production capabilities. It is easy to see that for each instant of time $t>t_{0}, K_{t}^{i}<x\left(f^{i}, v^{i}\right)$ and $f^{i}\left(K_{t}^{i}, 1\right)<f^{i}\left(x\left(f^{i}, v^{i}\right), 1\right)$. Therefore the inequality (2.2) is necessary for implementation of the $(i+1)$ th technology.

It is well known that for a model with a finite number of technologies all trajectories are bounded. For the model with discrete innovations a finite number of technologies can be introduced by time $t$, where $t=0,1, \ldots$, but the set of all technologies which can be utilized in the production process is infinite. In [2] we established the existence of trajectories of the model with discrete innovations on which consumption tends to infinity if the set $\left\{w\left(f^{i}, v^{i}\right): i \in I\right\}$ is unbounded and if

$$
\sup \left\{s^{(i+1) i}\left(f^{i}\left(x\left(f^{i}, v^{i}\right), 1\right)\right)^{-1}: i \in I\right\}<1 .
$$

In this paper we will establish the following result.

Theorem 2.2. Let

$$
\begin{gathered}
\sup \left\{w\left(f^{i}, v^{i}\right): i \in I\right\}=\infty, \\
\left(I_{0}^{0}, I_{n}^{0},\left(K_{0}^{i}, C_{0}^{i}\right)\left(I \in I_{0}^{0}\right)\right)
\end{gathered}
$$

be an initial state of the economy and let

$$
p=\sup \left\{i: i \in I_{0}^{0}\right\}, \quad K_{0}^{p}>0 .
$$

Then there exists a model trajectory

$$
\left(I_{0}^{t}, I_{n}^{t},\left(K_{0}^{i}, C_{0}^{i}\right)\left(i \in I_{0}^{t}\right)\right) \quad(t \in I),
$$


such that

$$
T^{-1} \sum_{t=1}^{T} \sup \left\{C_{t}^{i}: i \in I_{0}^{t}\right\} \longrightarrow \infty \quad \text { as } T \longrightarrow \infty
$$

and that for each $t>0, \sup \left\{C_{t}^{i}: i \in I_{0}^{t}\right\}>0$.

\section{Proof of Theorem 2.2}

We may assume without loss of generality that for any instant of time $t$ and any state of the model

$$
\left(\tilde{I}_{0}^{t}, \tilde{I}_{n}^{t},\left(\tilde{K}_{t}^{i}, \tilde{C}_{i}^{t}\right)\left(I \in \tilde{I}_{0}^{t}\right)\right)
$$

the following relation holds:

$$
\tilde{I}_{n}^{t}=\left\{\max \left\{i: i \in \tilde{I}_{0}^{t}\right\}+1\right\}
$$

In view of this assumption we omit below the notation $I_{n}^{t}$ in describing the state of the model. Clearly, we may also assume without loss of generality that

$$
I_{0}^{0}=\{p\}
$$

For $i \in I$ we set

$$
\begin{gathered}
w(i)=w\left(f^{i}, v^{i}\right), \quad h(i)=h\left(f^{i}, v^{i}\right), \quad x(i)=x\left(f^{i}, v^{i}\right), \\
\Lambda(i)=x\left(h\left(f^{i}, v^{i}\right) f^{i}, v^{i}\right) .
\end{gathered}
$$

Set $p_{0}=p$. By (2.4) there exists a strictly increasing sequence of integers $p_{i} \in I, i=0,1, \ldots$ such that

$$
w\left(p_{i+1}\right) \geq 2 w\left(p_{i}\right) \quad \forall i \in I
$$

For each $i \in I$ set

$$
\tilde{K}^{i}=\min \left\{4^{-1} \Lambda(i), K^{i}\right\}
$$

Let $i \in I$. Set

$$
\Gamma(i, 0)=\tilde{K}^{i}, \quad \Gamma(i, p+1)=v^{i} \Gamma(i, p)+f^{i}(\Gamma(i, p), 1), \quad p \in I
$$

It follows from (2.2) and (3.7) that there is a natural number $q(i)>2$ such that

$$
s^{(i+1) i}<f^{i}(\Gamma(i, q(i)-1), 1) .
$$

Lemma 3.1. Let $\tau \in I,\left(I_{0}^{\tau},\left(K_{\tau}^{i}, C_{\tau}^{i}\right)\left(i \in I_{0}^{\tau}\right)\right)$ be a state of the economy at time $\tau$ and let

$$
j=\max \left\{i: i \in I_{0}^{\tau}\right\}, \quad K_{\tau}^{j} \geq \tilde{K}^{j} .
$$


6 Existence of trajectories

Then there exists a model trajectory

$$
\left(I_{0}^{t},\left(K_{t}^{i}, C_{t}^{i}\right)\left(i \in I_{0}^{t}\right)\right) \quad(t=\tau, \ldots, \tau+q(j))
$$

such that

$$
\begin{gathered}
I_{0}^{\tau+q(j)}=I_{0}^{\tau} \cup\{j+1\}, \quad K_{\tau+q(j)}^{j+1}=K^{j+1}, \\
C_{t}^{j}>0, \quad t=\tau+1, \ldots, \tau+q(j) .
\end{gathered}
$$

Proof. Set

$$
l=s^{(j+1) j}\left(f^{j}(\Gamma(j, q(j)-1), 1)\right)^{-1} .
$$

For $t=\tau, \ldots, \tau+q(j)-2$ we set

$$
\begin{gathered}
I_{0}^{t+1}=I_{0}^{t}=I_{0}^{\tau}, \\
L_{t}^{i}=0, \quad i \in I_{0}^{\tau} \backslash\{j\}, \quad L_{t}^{j}=1, \\
C_{t+1}^{i}=0, \quad K_{t+1}^{i}=v^{i} K_{t}^{i}, \quad i \in I_{0}^{\tau} \backslash\{j\} . \\
K_{t+1}^{j}=v^{j} K_{t}^{j}+f^{j}\left(K_{t}^{j},(1+l) / 2\right), \\
C_{t+1}^{j}=f^{j}\left(K_{t}^{j}, 1\right)-f^{j}\left(K_{t}^{j},(1+l) / 2\right) .
\end{gathered}
$$

Clearly the states of the trajectory at times $t=\tau, \ldots, \tau+q(j)-1$ are well defined and

$$
C_{t}^{j}>0, \quad t=\tau+1, \ldots, \tau+q(j)-1 .
$$

We show that

$$
f^{j}\left(K_{\tau+q(j)-1}^{j}, 1\right)>s^{(j+1) j} .
$$

First we show by induction that for $t=0, \ldots, q(j)-1$,

$$
K_{t+\tau}^{j} \geq \Gamma(j, t)(1+l) / 2
$$

In view of (3.9) and (3.6) the inequality (3.16) is valid for $t=0$. Assume that (3.16) holds with an integer $t$ satisfying

$$
0 \leq t<q(j)-1 \text {. }
$$

It follows from (3.13), (3.16) and (3.7) that

$$
\begin{aligned}
K_{t+1+\tau}^{j} & =v^{j} K_{t+\tau}^{j}+f^{j}\left(K_{t+\tau}^{j},(1+l) / 2\right) \\
& \geq v^{j} \Gamma(j, t)(1+l) / 2+((1+l) / 2) f^{j}(\Gamma(j, t), 1) \\
& \geq((1+l) / 2) \Gamma(j, t+1) .
\end{aligned}
$$


Therefore (3.16) is true for all $t=0, \ldots, q(j)-1$ and

$$
K_{\tau+q(j)-1}^{j} \geq((1+l) / 2) \Gamma(j, q(j)-1) .
$$

By (3.19), (3.12) and (3.8),

$$
\begin{aligned}
f^{j}\left(K_{\tau+q(j)-1}^{j}, 1\right) & \geq f^{j}\left(2^{-1}(1+l) \Gamma(j, q(j)-1), 1\right) \\
& >2^{-1}(1+l) f^{j}(\Gamma(j, q(j)-1), 1)>s^{(j+1) j} .
\end{aligned}
$$

Define the state of the economy at time $\tau+q(j)$ as follows:

$$
\begin{gathered}
I_{0}^{\tau+q(j)}=I_{0}^{\tau} \cup\{j+1\}, \quad L_{\tau+q(j)-1}^{i}=0, \quad i \in I_{0}^{\tau} \backslash\{j\}, \\
L_{\tau+q(j)-1}^{j}=1, \quad C_{\tau+q(j)}^{i}=0, \quad K_{\tau+q(j)}^{i}=v^{i} K_{\tau+q(j)-1}^{i}, \quad i \in I_{0}^{\tau} \backslash\{j\}, \\
K_{\tau+q(j)}^{j}=v^{j} K_{\tau+q(j)-1}^{j}, \quad C_{\tau+q(j)}^{j}=f^{j}\left(K_{\tau+q(j)-1}^{j}, 1\right)-s^{(j+1) j}, \\
K_{\tau+q(j)}^{j+1}=K^{j+1}, \quad C_{\tau+q(j)}^{j+1}=0 .
\end{gathered}
$$

It is easy to see that the state of the economy at time $\tau+q(j)$ is well defined. Lemma 3.1 is proved.

Lemma 3.2. Let $\tau, k \in I$ and let

$$
\left(I_{0}^{t},\left(K_{t}^{i}, C_{t}^{i}\right)\left(i \in I_{0}^{t}\right)\right) \quad(t=0, \ldots, \tau)
$$

be a model trajectory. Assume that

$$
\begin{gathered}
p_{k}=\max \left\{i: i \in I_{0}^{\tau}\right\}, \\
K_{\tau}^{p_{k}} \geq 4^{-1} \Lambda\left(p_{k}\right) .
\end{gathered}
$$

Then there exists a model trajectory

$$
\left(I_{0}^{t},\left(K_{t}^{i}, C_{t}^{i}\right)\left(i \in I_{0}^{t}\right)\right) \quad(t=\tau, \ldots, \tau+S)
$$

with an integer $S \geq 1$ and an integer $T \in[1, S)$ such that

$$
\begin{gathered}
\max \left\{i: i \in I_{0}^{\tau+S}\right\}=p_{k+1}, \\
K_{\tau+S}^{p_{k}+1} \geq 4^{-1} \Lambda\left(p_{k+1}\right), \\
\sup \left\{C_{t}^{i}: i \in I_{0}^{t}\right\}>0, \quad t=\tau+1, \ldots, \tau+S, \\
C_{t}^{p_{k}} \geq 4^{-1} w\left(p_{k}\right), \quad t=\tau+1, \ldots, \tau+T, \\
Q^{-1} \sum_{t=1}^{Q} \sup \left\{C_{t}^{i}: i \in I_{0}^{t}\right\} \geq 16^{-1} w\left(f^{p_{k}}, v^{P_{k}}\right)
\end{gathered}
$$

for each $Q=T+\tau+1, \ldots, \tau+S$. 
8 Existence of trajectories

Proof. Set $a_{0}=K^{p_{k}+1}$ and

$$
a_{t+1}=v^{p_{k+1}} a_{t}+f^{p_{k+1}}\left(a_{k}, 1\right) h\left(f^{p_{k+1}}, v^{p_{k+1}}\right)
$$

for $t \in I$. Clearly

$$
\lim _{t \rightarrow \infty} a_{t}=\Lambda\left(p_{k+1}\right)
$$

There is a natural number $\tau_{0}$ such that

$$
a_{\tau_{0}} \geq 2^{-1} \Lambda\left(p_{k+1}\right) \text {. }
$$

Set

$$
\begin{gathered}
\bar{q}=\sum_{j=p_{k}}^{p_{k+1}-1} q(j), \\
T=\bar{q}+\tau_{0}+\tau, \quad S=T+\bar{q}+\tau_{0} .
\end{gathered}
$$

First we define the states of the trajectory at times $t=\tau+1, \ldots, \tau+T$ as follows:

$$
\begin{gathered}
I_{0}^{t}=I_{0}^{\tau}, \\
L_{t-1}^{i}=0, \quad i \in I_{0}^{\tau} \backslash\left\{p_{k}\right\}, \quad L_{t-1}^{p_{k}}=1, \\
K_{t}^{i}=v^{i} K_{t-1}^{i}, \quad C_{t}^{i}=0, \quad i \in I_{0}^{\tau} \backslash\left\{p_{k}\right\}, \\
K_{t}^{p_{k}}=v^{p_{k}} K_{t-1}^{p_{k}}+h\left(f^{p_{k}}, v^{p_{k}}\right) f^{p_{k}}\left(K_{t-1}^{p_{k}}, 1\right), \\
C_{t}^{p_{k}}=\left(1-h\left(p_{k}\right)\right) f^{p_{k}}\left(K_{t-1}^{p_{k}}, 1\right) .
\end{gathered}
$$

Using (3.24) and (3.31) we can show by induction that

$$
K_{t}^{p_{k}} \geq 4^{-1} \Lambda\left(p_{k}\right), \quad C_{t}^{p_{k}} \geq 4^{-1} w\left(p_{k}\right), \quad t=\tau+1, \ldots, \tau+T .
$$

In view of Lemma 3.1, (3.23), (3.31), and (3.32), there exists a model trajectory

$$
\left(I_{0}^{t},\left(K_{t}^{i}, C_{t}^{i}\right)\left(i \in I_{0}^{t}\right)\right) \quad\left(t=\tau+T, \ldots, \tau+T+\sum_{j=p_{k}}^{p_{k+1}-1} q(j)\right)
$$

such that

$$
\begin{gathered}
p_{k+1} \in I_{0}^{\tau+T+\bar{q}}, \quad K_{\tau+T+\bar{q}}^{p_{k+1}}=K^{p_{k+1}}, \\
\sup \left\{C_{t}^{i}: i \in I_{0}^{t}\right\}>0, \quad t=\tau+T+1, \ldots, \tau+T+\bar{q}, \\
p_{k+1}=\max I_{0}^{\tau+T+\bar{q}} .
\end{gathered}
$$


Now we define the states of the economy at time $t=\tau+T+\bar{q}+1, \ldots, \tau+S$ as follows:

$$
\begin{gathered}
I_{0}^{t}=I_{0}^{\tau+T+\bar{q}}, \\
L_{t-1}^{i}=0, \quad C_{t}^{i}=0, \quad K_{t}^{i}=v^{i} K_{t-1}^{i}, \quad i \in I_{0}^{\tau+T+\bar{q}} \backslash\left\{p_{k+1}\right\}, \\
L_{t-1}^{p_{k+1}}=1, \\
K_{t}^{p_{k+1}}=v^{p_{k+1}} K_{t-1}^{p_{k+1}}+h\left(p_{k+1}\right) f^{p_{k+1}}\left(K_{t-1}^{p_{k+1}}, 1\right), \\
C_{t}^{p_{k+1}}=\left(1-h\left(p_{k+1}\right)\right) f^{p_{k+1}}\left(K_{t-1}^{p_{k+1}}, 1\right) .
\end{gathered}
$$

(3.35), (3.29), (3.27), and (3.30) imply that

$$
K_{\tau+S}^{p_{k}+1}=a_{\tau_{0}} \geq 2^{-1} \Lambda\left(p_{k+1}\right) .
$$

In order to complete the proof of the lemma we need only to show that (3.26) is valid for $Q=\tau+1+T, \ldots, \tau+S$.

Assume that $\tau+T<Q \leq \tau+S$. It follows from (3.32) and (3.30) that

$$
\begin{aligned}
& Q^{-1} \sum_{t=1}^{Q} \sup \left\{C_{t}^{i}: i \in I_{0}^{t}\right\} \\
& \quad \geq(\tau+S)^{-1} \sum_{t=\tau+1}^{\tau+T} \sup \left\{C_{t}^{i}: i \in I_{0}^{t}\right\} \\
& \quad \geq(\tau+S)^{-1} 4^{-1} T w\left(p_{k}\right) \\
& \quad \geq(T+2 T)^{-1} T w\left(p_{k}\right) / 4 \geq 12^{-1} w\left(p_{k}\right)
\end{aligned}
$$

Lemma 3.2 is proved.

Completion of the proof of Theorem 2.2. Consider a sequence $\left\{b_{t}\right\}_{t=0}^{\infty} \subset R^{1}$ defined by

$$
b_{0}=K_{0}^{p}, \quad b_{t+1}=v^{p} b_{t}+h(p) f^{p}\left(b_{t}, 1\right), \quad t \in I .
$$

It is not difficult to see that

$$
\lim _{t \rightarrow \infty} b_{t}=\Lambda(p)
$$

There is an integer $t_{0} \geq 1$ such that

$$
b_{t_{0}} \geq 2^{-1} \Lambda(p)
$$

Define a trajectory

$$
\left(I_{0}^{t},\left(K_{t}^{i}, C_{t}^{i}\right)\left(i \in I_{0}^{t}\right)\right) \quad\left(t=0, \ldots, 4 t_{0}\right)
$$


For $t=1, \ldots, 4 t_{0}$ we set

$$
\begin{gathered}
I_{0}^{t}=I_{0}^{0}, \quad L_{t-1}^{i}=0, \quad C_{t}^{i}=0, \\
K_{t}^{i}=v^{i} K_{t-1}^{i}, \quad i \in I_{0}^{0} \backslash\{p\}, \quad L_{t-1}^{p}=1, \\
K_{t}^{p}=v^{p} K_{t-1}^{p}+h(p) f^{p}\left(K_{t-1}^{p}, 1\right), \quad C_{t}^{p}=(1-h(p)) f^{p}\left(K_{t-1}^{p}, 1\right) .
\end{gathered}
$$

It follows from (3.42) and (3.40) that

$$
\begin{aligned}
K_{t}^{p} \geq 2^{-1} \Lambda(p), & t=t_{0}, \ldots, 4 t_{0}, \\
C_{t}^{p} \geq 2^{-1} w(p), & t=t_{0}+1, \ldots, 4 t_{0} .
\end{aligned}
$$

Applying Lemma 3.2 by induction we construct a model trajectory

$$
\left(I_{0}^{t},\left(K_{t}^{i}, C_{t}^{i}\right)\left(I \in I_{0}^{t}\right)\right) \quad(t \in I)
$$

and strictly increasing sequences of natural numbers $\left\{T_{k}\right\}_{k=1}^{\infty},\left\{\tau_{k}\right\}_{k=0}^{\infty}$ such that

$$
\tau_{0}=4 t_{0}, \quad \tau_{k-1}<T_{k}<\tau_{k}, \quad k=1,2, \ldots
$$

and that for each integer $k \geq 0$ we have

$$
\begin{gathered}
\max \left\{i: i \in I_{0}^{\tau_{k}}\right\}=p_{k}, \\
K_{\tau_{k}}^{p_{k}} \geq 4^{-1} \Lambda\left(p_{k}\right), \\
\sup \left\{C_{t}^{i}: i \in I_{0}^{t}\right\}>0, \quad t=\tau_{k}+1, \ldots, \tau_{k+1}, \\
C_{t}^{p_{k}} \geq 4^{-1} w\left(p_{k}\right), \quad t=\tau_{k}+1, \ldots, T_{k+1}, \\
Q^{-1} \sum_{t=1}^{Q} \sup \left\{C_{t}^{i}: i \in I_{0}^{t}\right\} \geq 16^{-1} w\left(p_{k}\right), \\
Q=T_{k+1}+1, \ldots, \tau_{k+1} .
\end{gathered}
$$

It follows from (3.46), (3.45) and (3.42) that

$$
\sup \left\{C_{t}^{i}: i \in I_{0}^{t}\right\}>0, \quad t \in I \backslash\{0\} .
$$

We will show that

$$
T^{-1} \sum_{t=1}^{T} \sup \left\{C_{t}^{i}: i \in I_{0}^{T}\right\} \longrightarrow \text { as } T \longrightarrow \infty .
$$

By (3.45) and (3.43) for each integer $T \in\left[2 t_{0}, \tau_{0}\right]$

$$
T^{-1} \sum_{t=1}^{T} \sup \left\{C_{t}^{i}: i \in I_{0}^{t}\right\} \geq\left(4 t_{0}\right)^{-1} t_{0} 2^{-1} w\left(p_{0}\right) \geq 8^{-1} w\left(p_{0}\right) .
$$

Let $k \geq 0$ be an integer and assume that an integer

$$
T \in\left(\tau_{k}, T_{k+1}\right] \text {. }
$$


It follows from (3.46), and (3.49) that

$$
\begin{aligned}
T^{-1} \sum_{t=1}^{T} \sup \left\{C_{t}^{i}: i \in I_{0}^{t}\right\} \\
\quad=T^{-1}\left[\sum_{t=1}^{\tau_{k}} \sup \left\{C_{t}^{i}: i \in I_{0}^{t}\right\}+\sum_{t=\tau_{k}+1}^{T} \sup \left\{C_{t}^{i}: i \in I_{0}^{t}\right\}\right] \\
\quad \geq T^{-1}\left[\tau_{k} \sum_{t=1}^{\tau_{k}} \sup \left\{C_{t}^{i}: i \in I_{0}^{t}\right\} / \tau_{k}+\left(T-\tau_{k}\right) 4^{-1} w\left(p_{k}\right)\right] \\
\quad \geq T^{-1}\left[\tau_{k} / 16^{-1} w\left(p_{k}\right)+\left(T-\tau_{k}\right) 4^{-1} w\left(p_{k}\right)\right] \geq 16^{-1} w\left(p_{k}\right) .
\end{aligned}
$$

Together with (3.46) this implies that for each natural number $T \in\left(\tau_{k}, \tau_{k+1}\right]$

$$
T^{-1} \sum_{t=1}^{T} \sup \left\{C_{t}^{i}: i \in I_{0}^{t}\right\} \geq 16^{-1} w\left(p_{k}\right) .
$$

Since $w\left(p_{k}\right) \rightarrow \infty$ as $k \rightarrow \infty$, the inequality above completes the proof of the theorem.

\section{References}

[1] V. L. Makarov, On dynamic models of economy and development of ideas of L.V. Kantorovich, Economics and Mathematical Methods 23 (1987), 10-24.

[2] A. J. Zaslavski, On a model of economic dynamics with discrete innovations, Economics and Mathematical Methods 25 (1989), 679-684.

[3] _ـ Discrete innovations in dynamic models of economy, Siberian Mathematical Journal 31 (1990), 47-59.

[4] - Optimization of the growth rate in models with discrete innovations, Siberian Mathematical Journal 32 (1991), 43-50.

[5] __ Asymptotic behavior of paths of a model with discrete innovations, Siberian Mathematical Journal 33 (1992), no. 4, 608-617.

[6] _ On a class of dynamical systems arising in mathematical economics. I, Dynamic Systems and Applications 7 (1998), no. 2, 215-236.

[7] __ Allocations of labour resources on trajectories for the model with discrete innovations, Advances in Mathematical Economics, Vol. 2, Adv. Math. Econ., vol. 2, Springer, Tokyo, 2000, pp. 137-160.

[8] _ On a class of dynamical systems arising in mathematical economics. II, Dynamic Systems and Applications 9 (2000), no. 1, 15-35.

[9] Existence of trajectories with unbounded consumption for the Makarov model with discrete innovations, Nonlinear Analysis. Real World Applications 2 (2001), no. 2, 195-220.

Alexander J. Zaslavski: Department of Mathematics, The Technion - Israel Institute of Technology, 32000 Haifa, Israel

E-mail address: ajzasl@tx.technion.ac.il 